\title{
Desarrollo socioemocional en niños mexicanos: un estudio narrativo sobre la migración
}

\section{Socioemotional development in Mexican children: A narrative study regarding migration}

María de la Luz Pérez Padilla

Felipe Olmos Ríos

María Teresa Solorio Núñez

Universidad de Guadalajara

\section{Resumen}

Los niños como actores sociales también viven y observan la migración directa e indirectamente; de acuerdo con el contexto y su propio proceso psico-evolutivo, tienen una manera de narrar lo que saben y viven. La presente investigación hace un acercamiento a estos procesos socioemocionales a través de la narrativa y el dibujo de 652 niños de $3^{\circ}$ a $6^{\circ}$ de primaria de los estados de Zacatecas, Michoacán, Hidalgo, Jalisco y Yucatán. Se utilizó la investigación narrativa, donde ellos dibujaban su interpretación de la migración y relataban una historia al respecto. Asimismo, se analizó la información con categorías preestablecidas y emergentes, con base en ellas, se encontró que los niños expresan principalmente las emociones de tristeza y felicidad, asociadas a situaciones sociales donde hay peligro y amenaza ante la migración y donde se puede triunfar al vencer obstáculos y al lograr el objetivo de migrar. En concreto, se constató que estos niños tienen una consciencia social del otro, tanto de su dolor, alegría y las injusticias vividas.

Palabras clave: niños, narrativas, dibujos, migración, desarrollo socioemocional.

\section{Nota del autor}

María de la Luz Pérez Padilla, Felipe Olmos Ríos y María Teresa Solorio Núñez, Centro Universitario de los Lagos, Universidad de Guadalajara.

La correspondencia en relación con este artículo debe dirigirse a María de la Luz Pérez Padilla, Centro Universitario de los Lagos, Universidad de Guadalajara, Av. Enrique Díaz de León 144, Col. Paseos de la Montaña, C.P. 47460, Lagos de Moreno, Jalisco.

Correo electrónico: madelaluzperez@hotmail.com 


\begin{abstract}
Children as social actors live and observe migration directly and indirectly as well. According to the context and their psycho-evolutive process, they have a way to explain what they know and experience. This study sketches these socioemotional processes through narratives and drawings provided by 652 children from the third to the sixth grade in the states of Zacatecas, Michoacán, Hidalgo, Jalisco, and Yucatán. A narrative methodology was used, in which they drew their interpretation of migration and wrote a story related to it. The information was analyzed using pre-establish and emerging categories, finding that children express mainly sadness and happiness in association with dangerous and threatening social situations in whcih they perceive themselves as able to overcome obstacles in order to successfully migrate. Finally, we concluded that children have a social awareness of the pain, happiness, and injustices that are experienced in migratory processes.
\end{abstract}

Keywords: children, narratives, drawings, migration, socioemotional development.

La niñez es un período crítico para desarrollar habilidades socioemocionales. Aunque el niño posee una cierta predisposición por su temperamento, la interacción constante con su entorno dará lugar a manifestaciones sociales resultado de dicha interacción (Oros \& Fontana, 2015). Visto lo anterior, el presente trabajo se enfoca en el desarrollo socioemocional en los niños, manifestado en su experiencia con el fenómeno de la migración. Para ello, se define qué es lo socio-emocional, después se mencionan algunos aspectos del desarrollo socioemocional en los niños $\mathrm{y}$, por último, se explica la experiencia migratoria de los niños mediante algunos estudios previos.

\section{Lo socioemocional}

Los factores social y emocional son centrales en el desarrollo de la infancia y se enfatizan en este trabajo, sin embargo, primero es necesario aclarar qué se entiende por éstos. Definir lo que es una emoción parece ser una tarea compleja por las diversas definiciones que existen sobre ésta. Para este estudio, se ha optado por incluir la definición de Palermo, Guerrero, Gómez y Carpi (2006), ya que en su estudio, después de analizar la convergencia y discrepancia sobre las distintas definiciones propuestas por diversos autores, proponen un término de emociones que trata de integrar elementos comunes:

Procesos episódicos que, licitados por la presencia de algún estímulo o situación interna o externa, que ha sido evaluada y valorada como potencialmente capaz de producir un desequilibrio en el organismo, dan lugar a una serie de cambios o respuestas en los planos subjetivo, cognitivo, fisiológico y motor expresivo; cambios que están íntimamente relacionados con el mantenimiento del 
equilibrio, esto es: con la adaptación de un organismo a las condiciones específicas del medio ambiente (p. 21).

En esta definición se incluyen elementos cognitivos, al indicar que las personas evalúan y valoran los estímulos percibidos de acuerdo con la codificación que tienen respecto a si la situación presentada es agradable o amenazante.

\section{EI desarrollo socioemocional en los niños}

Desde el nacimiento, niños y niñas se desarrollan en contextos de interacción social en los que las emociones regulan las relaciones. De esta manera, durante los primeros años de vida, el menor aprenderá a manifestar sus propias emociones, a percibir las de los demás y a responder ante ellas, tratando de controlar sus propias emociones. Por consiguiente, su forma de aprender y el cómo se desarrolle emocionalmente, influirán de manera directa en su calidad de vida y bienestar (Heras, Cepa \& Lara, 2016). Por lo tanto, las emociones que vive el niño también tienen un componente de aprendizaje social en esta interacción. De acuerdo a Ortiz, López-Sánchez, Fuentes y Etxebarria (2014), así como Reeve (2004), lo social y afectivo implica un desarrollo evolutivo donde el niño va incorporando aquello que vive y socializa en su contexto para formar vínculos afectivos, aprender costumbres, roles, conductas $\mathrm{y}$ adquirir normas y valores del grupo al que pertenece.

Los niños de entre 8 y 12 años de edad se encuentran en los periodos concreto ( 7 a 12 años) y formal (12 años en delante) propuestos por Piaget (Papalia, Wendkons \& Duskin, 2009). Tienen la capacidad de mirar al otro y reconocer lo que le pasa, incluso tratan de sentir y pensar lo que el otro está experimentando, a esto se le llama empatía (Garaigordobil \& Maganto, 2011).

Según Rodas (2016), "la empatía es la respuesta afectiva-cognitiva, por parte del individuo que observa las vivencias de otras personas, activada por el estado de necesidad, influyendo en la manifestación de la percepción y valoración que tenga el individuo observador del bienestar de esa otra persona" (p. 10). Tal como lo indica la definición, se requiere un componente cognitivo para colocarse en la perspectiva del otro; tal componente se irá desarrollando con la maduración y estimulación. Empero, a su vez pervive en este proceso un elemento emocional necesario para que el niño pueda distinguir emociones en el otro y también sea capaz de sentirlas desde su propia perspectiva, aunque con apego a la experiencia de quien observa. Por supuesto, esta capacidad empática tiene una influencia social. Tal relación entre la emoción y la cognición es llamada por algunos autores Inteligencia Emocional (IE), ésta permite el funcionamiento adaptativo del individuo en el medio donde se relaciona (Arias, Morales, Nouvilas \& Martínez, 2012).

Oros y Fontana (2015), comentan que los niños empáticos, cuyas respuestas afectivas son acordes con la situación manifiesta, que reconocen su sentir y cuentan con una alta 
regulación empática, son más propicios a poner en acción habilidades adecuadas socialmente.

Por su parte, Erick Erikson propuso que en la etapa psicosocial del desarrollo entre los 6 y 12 años, los niños tienen la disyuntiva entre la laboriosidad y la inferioridad (Bordignon, 2005). Los niños en esta etapa buscan hacer cosas, planearlas, compartir con otros, desarrollar $\mathrm{y}$ reconocer sus habilidades para no sentirse incapaces o inferiores. Erikson, en su teoría del desarrollo psicosocial, recalca la importancia del aspecto social en el desarrollo del niño. Las influencias sociales y culturales que recibe cualquier persona a lo largo de su proceso de desarrollo van moldeando su patrón conductual básico, haciendo que se interiorice aquello que es socialmente aceptable, aquello que es injusto, entre otros elementos (Palmero Cantero et al., 2006). Somos seres sociales, por ende, existe una influencia bidireccional entre el niño y el medio en que se desenvuelve.

En cuanto a la percepción de los niños sobre el medio social que les rodea, Kohlberg y Hers (1977), comentan que el desarrollo moral entre los 9 y 12 años se encuentra en una etapa convencional. Es decir, los niños de 9 y 10 años juzgarán un acto como bueno o malo cuidando la relación con sus cercanos; un acto es bueno si le ayuda a tener una buena relación con el otro. Para los niños de 11 a 12 años, un acto es juzgado como correcto o incorrecto dependiendo de si altera o no el orden social, por lo cual los niños tendrán una opinión sobre la migración de acuerdo con su propia experiencia y etapa madurativa.

\section{Los niños y su experiencia migratoria}

Para quienes viven la migración de manera directa, es decir, migrando ellos mismos, o de manera indirecta, a través de un familiar cercano, sus vidas se ven influenciadas por esta situación que afecta todo el sistema familiar (Ramos, 2012). En cuanto al efecto de la migración en los niños, se ha encontrado que puede provocar trastornos, violencia, abuso de substancias y desórdenes de la conducta como la psicosis o trastornos asociados al proceso de adaptación (Gamarra et al., 2006; García \& Sanz, 2002; Maldonado, 2005, citados por Vera, 2009; Esquivel, 2010; Guzmán-Carrillo \& GonzálezVerduzco, 2013).

Estos problemas de salud mental se dan, principalmente, por el proceso de reajuste que llevan a cabo quienes viven esta experiencia. Esquivel (2010) menciona que adaptarse a múltiples cambios puede ser un factor de riesgo que genere psicopatologías; por ello, en el mundo de los sentimientos y emociones del infante, la familia juega un rol fundamental (Gloria, 1999). Lo que suceda dentro de ésta, afectará a todos sus integrantes.

El caso de la pérdida de un miembro de la familia a causa de la migración, implica un duelo familiar con posibles consecuencias tanto sociales como psicológicas. Sin embargo, como la pérdida es ambigua (Falicov, 2001), de alguna manera complica el proceso de duelo, ya que la 
familia tiende a reorganizarse constantemente (García-Ledesma \& Suárez-Castillo, 2007; López-Pozos, 2009).

Piras (2016) comenta que los sentimientos generados en los hijos o familiares de los migrantes, dependerán de las razones de la ausencia y de si los niños ya lograron elaborar su duelo, aunque el duelo es complejo por su ambigüedad (Boss, 2001). Por esta misma razón, las emociones generadas por la partida del migrante pueden ser contradictorias: por un lado, experimentar depresión, tristeza, abandono (López-Pozos, 2009), así como rencory, por otro, sentir, orgullo, satisfacción y agradecimiento (Piras, 2016; Ojeda García, 2014).

Tanto los migrantes como los niños, hijos o familiares de migrantes, viven experiencias que ponen a prueba su capacidad de resistencia, de adaptación y de fortalecerse ante la adversidad. De alguna forma, la experiencia migratoria es una experiencia de resiliencia. "La resiliencia hace referencia a aquello que pone de manifiesto la capacidad que el individuo tiene para enfrentar con éxito la adversidad y la fuerza flexible que permite resistir y rehacerse después de una condición adversa" (Castañer, 2017, p. 8). Para el caso de los niños, el poder sobreponerse a la ausencia del familiar que migra y asumir nuevos roles en el sistema familiar, definitivamente es una oportunidad de ejercer y desarrollar resiliencia. Autores como Gortberg (2008) mencionan que, a partir de los 9 años de edad, los niños ya son capaces de promover su propia resiliencia y buscar mayor ayuda externa, un proceso que puede ser promovido a lo largo de la vida.

Este trabajo tiene como propósito ofrecer un acercamiento para ver y escuchar a niños desde su propia perspectiva, al permitir conocer sus percepciones y emociones ante el fenómeno de la migración. Evidenciar estos procesos evolutivos del desarrollo socioemocional, imprime un aporte al conocimiento tanto de la psicología del desarrollo y nos allega a la comprensión de la mirada de los niños sobre su experiencia con la migración. Por ello, se intentará tener un acercamiento a su subjetividad a través de esas historias narradas, al igual que los dibujos producidos por niños de diferentes regiones geográficas de México.

\section{Método}

\section{Contexto de la investigación}

Este trabajo se deriva de una colaboración entre diferentes universidades de México que participan en la Cátedra CUMEX: Universidad Autónoma de Yucatán (UADY), Universidad Autónoma de Zacatecas (UAZ), Universidad Michoacana de San Nicolás de Hidalgo (UMNSH) y el Centro Universitario de los Lagos de la Universidad de Guadalajara (UdeG), tres de éstas están insertas en la región centro occidente, Hidalgo, en el centro, y Yucatán, en el sur.

\section{Diseño de investigación}

Este es un estudio cualitativo, con base en la narrativa como método de recolección y de 
análisis de la información. Considera que todas las formas de investigación narrativa muestran un constructo social que es influido por cuestiones históricas, socioculturales y políticas (Serry \& Liamputtong, 2013).

\section{Participantes}

Los participantes del estudio fueron niños de entre 8 y 12 años, que cursaban 3, 4, 5 y 6 grado en escuelas primarias públicas, pertenecientes a los cuatro estados mencionados.

\section{Técnicas de recolección y análisis}

Se utilizó la narrativa como método de recolección de información, siguiendo el procedimiento sugerido por Strauss (1970): recolectar datos, codificar y hacer una reflexión analítica de lo encontrado. Se hicieron preguntas abiertas sobre por qué se daba la migración, cómo era ésta para ellos (buena o mala) y por qué.

Tabla 1

Distribución pro estado donde viven, sexo, rango de edad y grado escolar de los participantes

\begin{tabular}{lcccccccccc}
\hline Estado & Niños & Niñas & $\begin{array}{c}\text { Sin } \\
\text { Dato }\end{array}$ & $\begin{array}{c}\mathbf{8}-\mathbf{9} \\
\text { años }\end{array}$ & $\begin{array}{c}\mathbf{1 0 - 1 1} \\
\text { años }\end{array}$ & $\begin{array}{c}\mathbf{1 2 - 1 3} \\
\text { años }\end{array}$ & $\begin{array}{c}\text { 3er } \\
\text { grado }\end{array}$ & $\begin{array}{c}\mathbf{4}^{\mathbf{0}} \\
\text { grado }\end{array}$ & $\begin{array}{c}\mathbf{5}^{\mathbf{2}} \\
\text { grado }\end{array}$ & $\begin{array}{c}\mathbf{6}^{\circ} \\
\text { grado }\end{array}$ \\
\hline Michoacán & 56 & 59 & & 54 & 56 & 5 & 30 & 32 & 31 & 22 \\
Zacatecas & 38 & 48 & & 9 & 51 & 26 & & 29 & 29 & 28 \\
Hidalgo & 55 & 57 & 2 & 53 & 35 & 26 & 32 & 49 & & 33 \\
Yucatán & 71 & 92 & & 50 & 65 & 48 & 36 & 38 & 37 & 52 \\
Jalisco & 107 & 67 & & 75 & 91 & 8 & & 84 & 90 & \\
Subtotales & 327 & 323 & 2 & 241 & 298 & 113 & 98 & 232 & 187 & 135 \\
Total & 652 & & & & & & & & & \\
\hline
\end{tabular}

Asimismo, se utilizó el dibujo como herramienta de recolección de información, ya que este medio lo utiliza el niño para acercarse a su realidad vivida, le permite expresar ideas, sentimientos y emociones, tanto de experiencias internas como de su alrededor; ésta es su forma natural de comunicación (Puleo, 2012).

Tanto las narraciones, las respuestas a las preguntas abiertas, como los dibujos se organizaron, se describieron y se transcribieron. Posteriormente, se analizó la información utilizando el programa Atlas $\mathrm{Ti}$, donde se determinaron las categorías a partir del análisis (López \& Scandroglio, 2007). Se organizó la información según dos categorías generales: emociones asociadas a la migración y desarrollo social manifiesto en los significados de la migración. Algunos dibujos y narraciones 
representativos fueron seleccionados para mostrar esas diferencias entre grupos de edad y el contenido de lo socioemocional de los mismos. La tabla 2 muestra las categorías de análisis.

\section{Resultados}

Las categorías de análisis de las narrativas de los niños se clasifican en emociones asociadas a la migración (tristeza, felicidad, sentido de justicia y de sacrificio) y desarrollo social manifiesto en los significados de la migración. Estas dos grandes categorías con sus respectivas subcategorías se organizaron por rangos de edad, grupo de 8 a 9 años, grupo de 10 a 11 años y grupo de 12 años.

\section{Emociones asociadas a la migración} Tristeza hacia sí mismo.

En las categorías de tristeza, el niño expresa explícita o implícitamente la tristeza que experimenta al tener un familiar cercano que se va, al saber de las situaciones difíciles que viven o pueden vivir estos familiares, tanto en el cruce como en el establecimiento, pero su focalización principal es sobre lo que ellos han perdido, lo que experimentan, de tal forma que refieren sentimientos de tristeza, abandono, soledad, y de extrañar mucho a quien se ha marchado.

Tabla 2

Categorías de análisis y sus definiciones

\begin{tabular}{ll}
\hline Categoría & \multicolumn{1}{c}{ Definición } \\
\hline Emociones asociadas a la migración & $\begin{array}{l}\text { Emoción que experimentan los niños a través de las historias de los personajes } \\
\text { donde ellos están involucrados } \\
\text { Emoción experimentada por los niños ante los personajes de su historia por las } \\
\text { Tristeza hacia sí mismo } \\
\text { Tristeza hacia los otros } \\
\text { Felicidad por }\end{array} \quad \begin{array}{l}\text { Engloba la emoción que experimentan ellos y los personajes de las historias } \\
\text { cuando logran reunirse con sus familiares, ya sea por un periodo de tiempo } \\
\text { corto (solo visitas) o definitivamente }\end{array}$ \\
\hline Felicidad por logro & $\begin{array}{l}\text { Refleja la emoción que experimentan los personajes cuando logran realizar } \\
\text { algo bueno para ellos y sus familias (cruzar la frontera, encontrar trabajo, etc.) }\end{array}$ \\
\hline Desarrollo social manifiesto en los significados de la migración \\
\hline $\begin{array}{l}\text { Sentido de justicia } \\
\text { Sentido de sacrificio } \\
\text { del migrante por el } \\
\text { bienestar de la familia }\end{array}$ & $\begin{array}{l}\text { Hace referencia a cómo los niños se percatan de injusticias que se dan ante la } \\
\text { migración y las plasman en sus historias. }\end{array}$ \\
\hline
\end{tabular}




\section{Tabla 3}

Expresiones de tristeza por verse afectados directamente por la migración

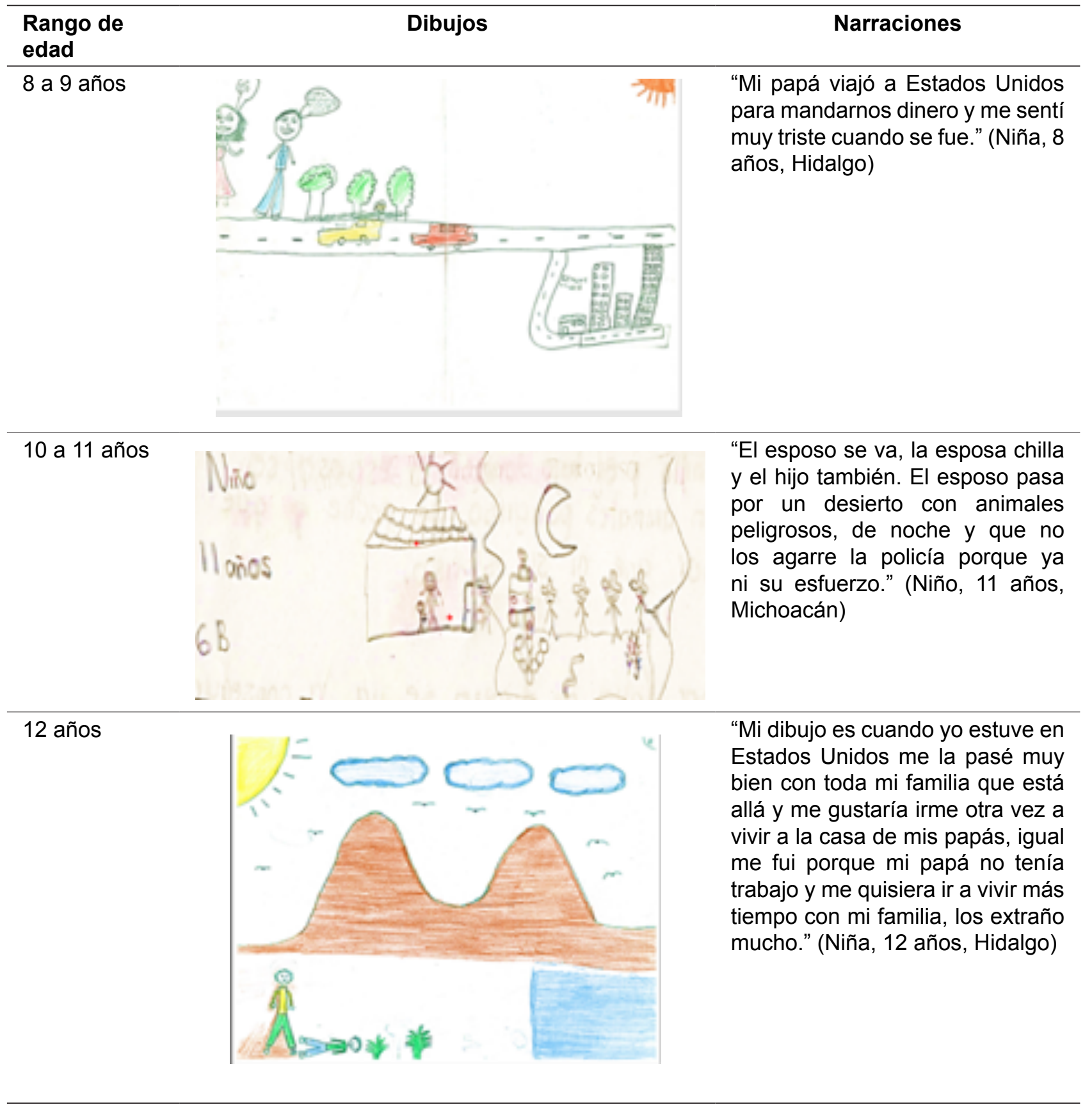

\section{Tristeza hacia los otros.}

Los niños perciben la tristeza de los otros. cualquier familiar del migrante que vive una situación difícil al quedarse sin el que se va.

Esos otros pueden ser las esposas, los hijos o 
Tabla 4

Manifestaciones de tristeza por la situación de migrantes y sus familias

\begin{tabular}{|c|c|c|}
\hline $\begin{array}{l}\text { Rango de } \\
\text { edad }\end{array}$ & Dibujos & Narraciones \\
\hline 8 a 9 años & & $\begin{array}{l}\text { "El papá se va a E. U., su esposa } \\
\text { y su hija se ponen tristes." (Niña, } \\
9 \text { años, Michoacán) }\end{array}$ \\
\hline
\end{tabular}

10 a 11 años

12 años

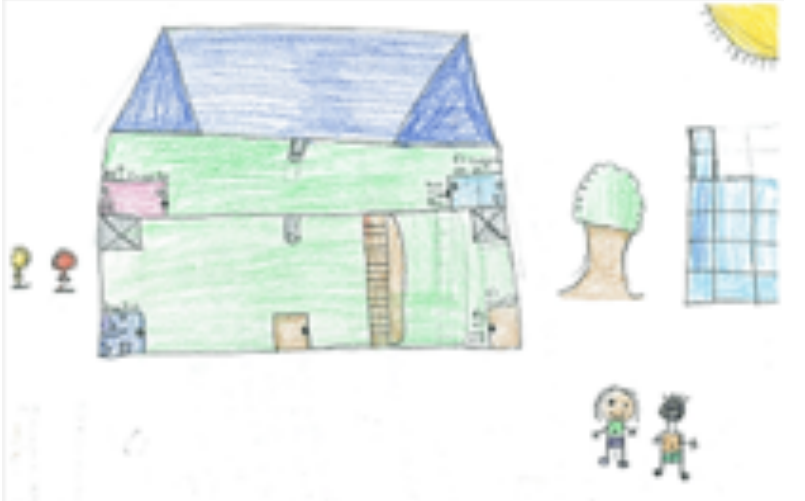

"Había una vez una niña que se iba a migrar y su amiga no quería porque la iba a dejar sola pero la niña sí se fue, su amiga se puso muy triste." (Niña, 10 años, Hidalgo)
"Hace mucho tiempo dos hermanos no tenían trabajo en su estado y como no tenían para comer decidieron irse al otro lado, pero pasaron de mojados, es muy doloroso porque a medio camino ya casi llegando a la frontera tienen mucho peligro de que los agarre la migra y te regrese, después tienen mucho peligro de las víboras, alacranes o tarántulas y después muriéndose de sed. No podrías llegar a Estados Unidos, no hagas eso y después de mucho pero mucho tiempo encontraron a los dos muchachos muertos fin." (Niña, 12 años , Hidalgo) 


\section{Felicidad ante la migración}

Felicidad ante el reencuentro.

En contraposición con la tristeza, los niños también refieren emociones de felicidad, de alegría. Éstas están asociadas a la reunificación de la familia, poder ver nuevamente al familiar que se fue. Poder tener un encuentro con él, ya sea temporal o permanente, es objeto de gran felicidad para los niños.

Tabla 5

Niños expresando felicidad por el reencuentro con familiares migrantes

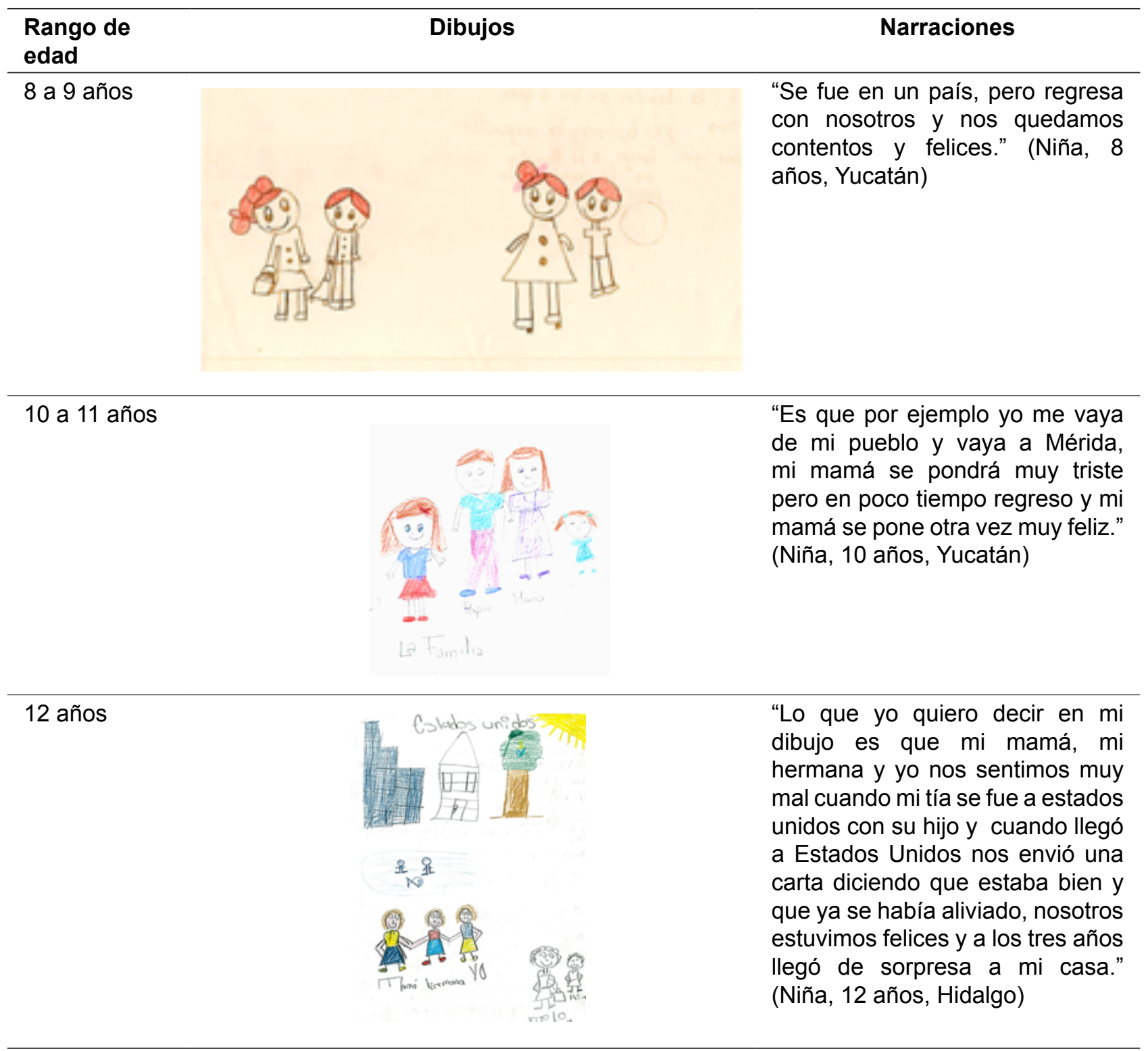




\section{Felicidad por el logro.}

Lafelicidad también estáasociadaallogro. Los niños expresan su alegría al mencionar el poder cumplir las metas planteadas por el migrante, el poder cruzar, sobrevivir a los peligros, lograr conseguir trabajo, lograr establecerse en el otro lado y prosperar económicamente.

Tabla 6

Niños expresando felicidad por el logro de algún migrante

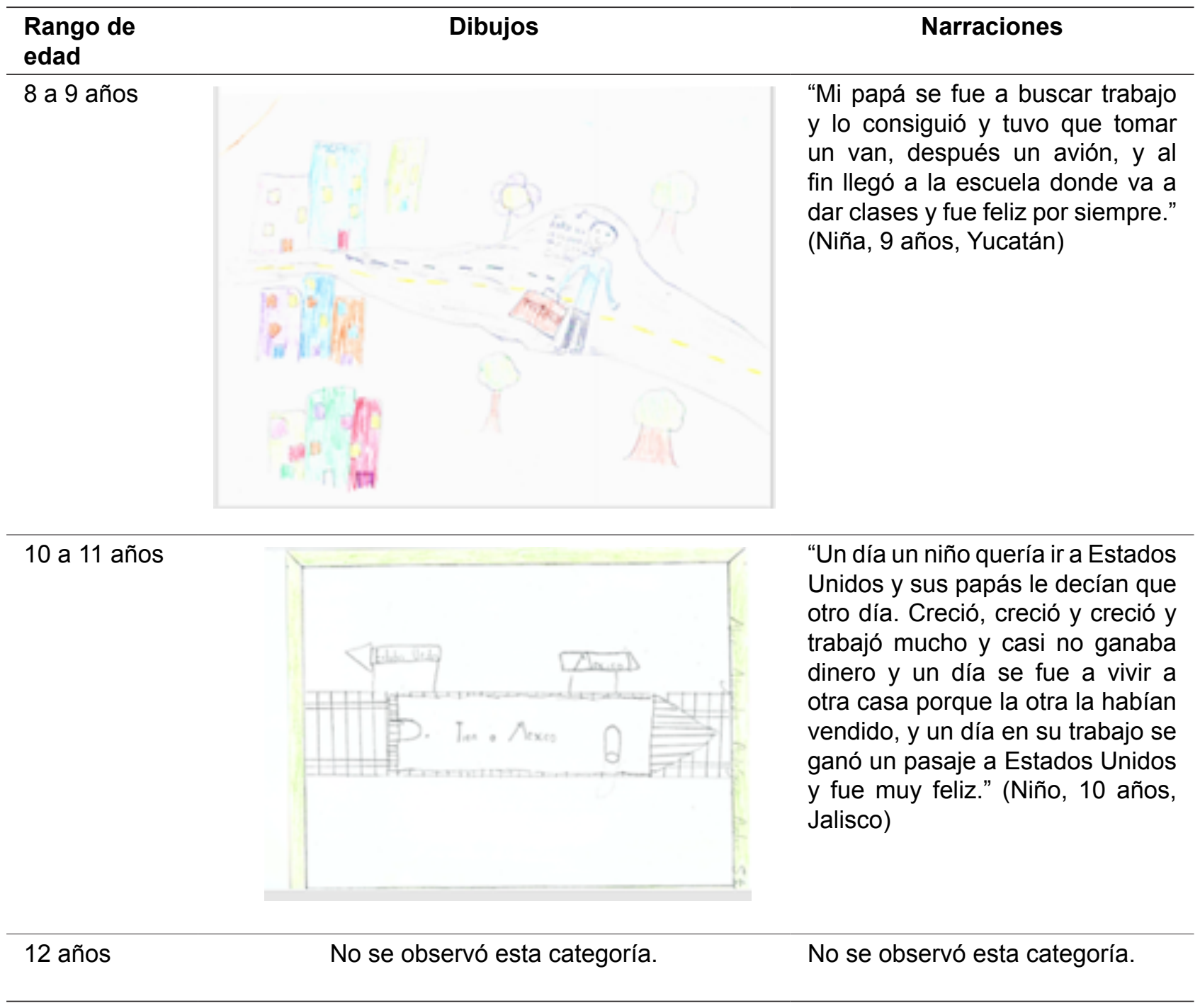


Desarrollo social manifiesto en los significados de la migración

\section{Migrar es amor y sacrificio.}

Cabe resaltar que los niños asocian las experiencias vividas por ellos y los migrantes ante la migración con alegrías y tristezas, como una muestra de amor y sacrificio. Mencionan que, aunque ellos o sus familiares que migran o los que se quedan sufran de dicha experiencia, ésta es una manera de manifestar amor por parte del migrante que se va, interpretan que tal circunstancia se da por el bien común.

\section{Tabla 7}

Niños expresando lo que para ellos significa migrar

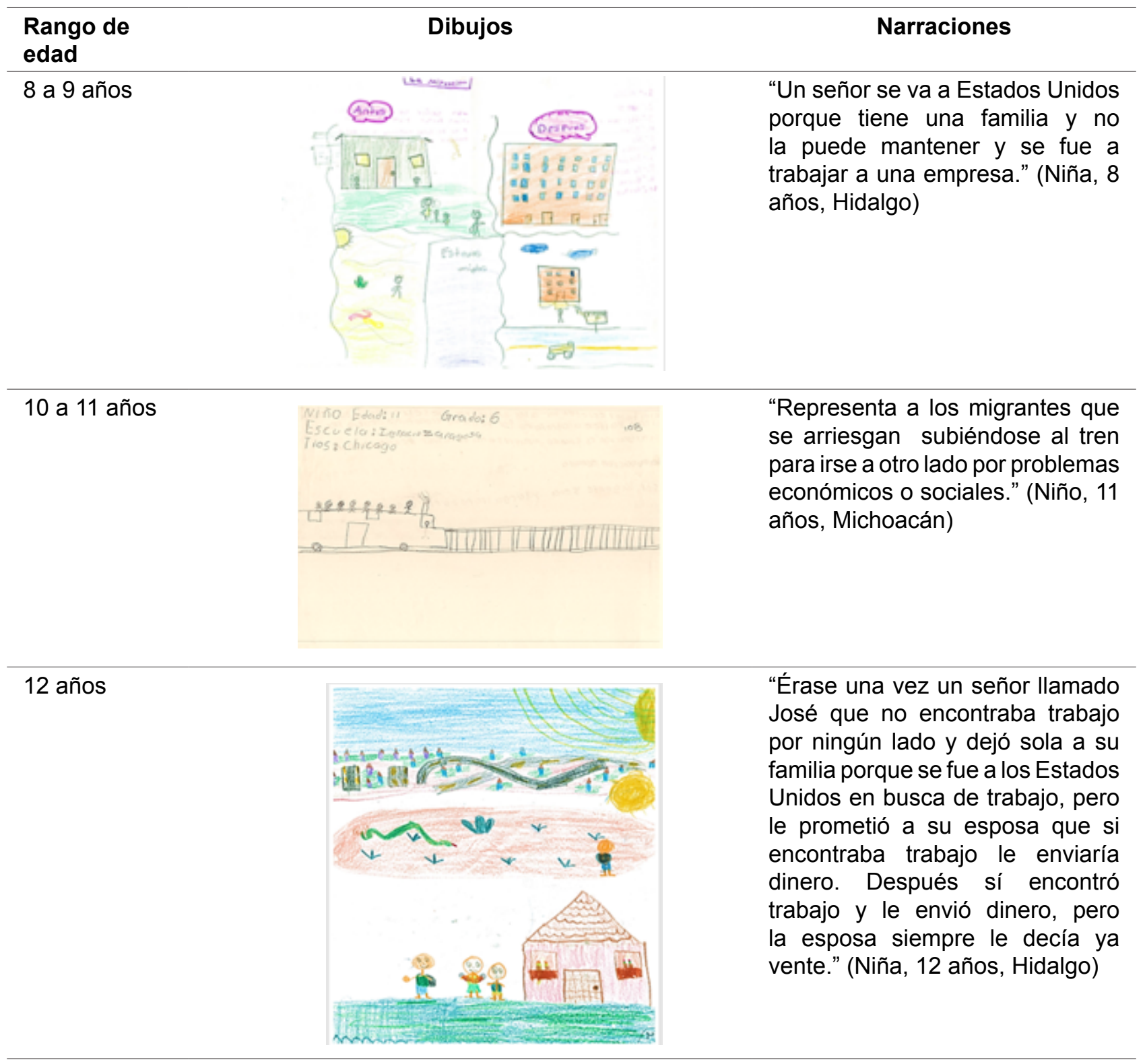


Conciencia social ante la discriminación y las injusticias

Tanto el sentido de sacrificio, de amor y de justicia que le dan los niños al proceso migratorio da cuenta de la conciencia social que han desarrollado. Asimismo, lo constata el cómo los niños reportan y perciben de manera muy clara cuando ocurren situaciones de injusticia y discriminación, temas principales que reconocen en sus historias, tal y como se muestra a continuación:

\section{Tabla 8}

Niños expresando su interpretación de la pobreza, las injusticias y la inequidad asociada a las migración

\begin{tabular}{|c|c|c|}
\hline $\begin{array}{l}\text { Rango de } \\
\text { edad }\end{array}$ & Dibujos & Narraciones \\
\hline 8 a 9 años & & $\begin{array}{l}\text { "Viven en pobreza y se mudan a } \\
\text { Estados Unidos para tener una } \\
\text { vida mejor." (Niño, } 9 \text { años, Jalisco) }\end{array}$ \\
\hline 10 a 11 años & & $\begin{array}{l}\text { "Es injusta porque los policías les } \\
\text { pegan, ellos no tienen la culpa, } \\
\text { hasta las mamás se van y se } \\
\text { llevan a sus hijos, pero a veces } \\
\text { los dejan en la frontera. Está muy } \\
\text { bien que las señoras les den de } \\
\text { comer a los migrantes y tengan en } \\
\text { donde descansar." (Niña, } 11 \text { años, } \\
\text { Michoacán) }\end{array}$ \\
\hline
\end{tabular}

12 años

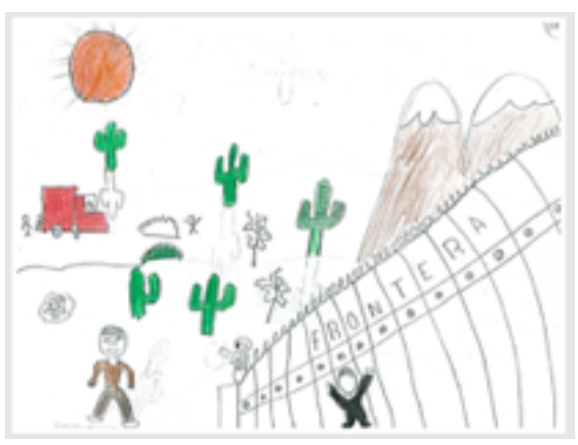

"Esto es lo que nos orillan a hacer por falta de empleo, atención médica y por la delincuencia que hay en México por eso los que cruzan la frontera pasan porque piensan que va a haber más oportunidades de trabajo y más recursos económicos." (Niño, 12 años, Hidalgo) 


\section{Integración del análisis de categorías}

La figura 1 busca integrar las categorías y su relación con las situaciones migratorias desde las diferentes experiencias narradas por los niños en sus historias y dibujos.

Como se puede apreciar en la figura 1, los niños experimentan diferentes emociones, principalmente tristeza y alegría. La tristeza es provocada por el hecho de que algunos niños perciben el alejamiento de sus familiares como un acto de abandono. Asimismo, suelen experimentar tristeza hacia la situación de migrar $\mathrm{y}$ hacia quienes migran debido a que reconocen hechos violentos donde hay discriminación.
Además de experimentar sentimientos de tristeza, también sienten felicidad. El sentimiento de felicidad parte de la percepción del sacrificio que hace el familiar que migra por el bienestar de la familia, lo cual ven como un logro y como una muestra de amor y cariño hacia ellos y su familia; así también, sienten felicidad por la reunificación cuando el familiar vuelve. Ambas emociones denotan la existencia de una conciencia social y empatía por parte del niño; puede reconocer lo que le sucede a él y lo que les sucede a los otros cuando se da la migración de algún familiar cercano.

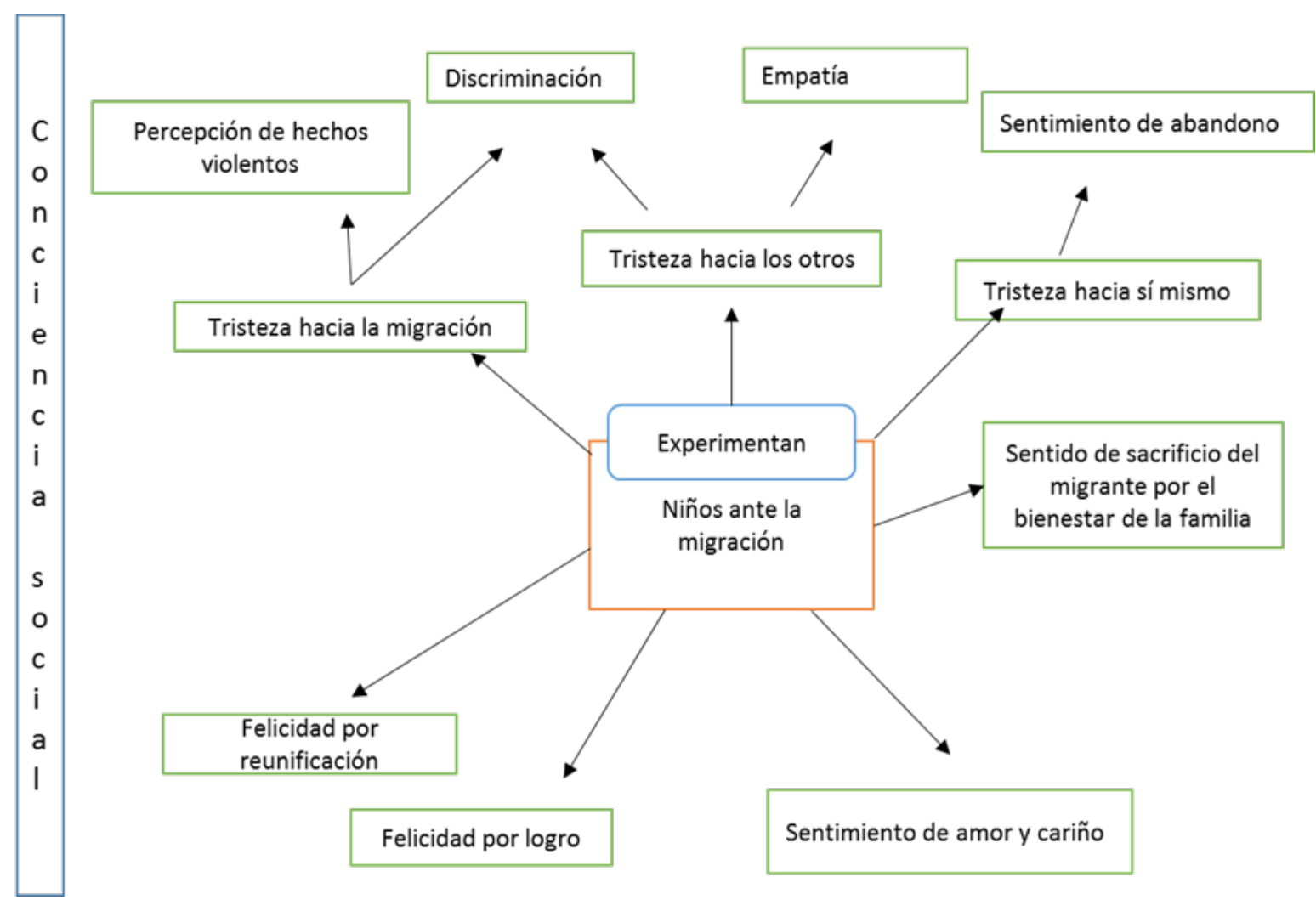

Figura 1. Integración del análisis de categorías preestablecidas y emergentes 


\section{Discusión y conclusiones}

Tal como lo dicen Ortiz, López-Sánchez, Fuentes y Etxebarria (2014) y Reeve (2004), lo social y afectivo está estrechamente ligado en el desarrollo del niño. En los niños de este estudio, su contexto y sus propias narrativas dan cuenta de ese proceso de socialización que están viviendo en torno a la migración y las adecuaciones que viven quienes experimentan la migración directa o indirectamente, ya sea porque algún familiar cercano migró o porque alguien de su comunidad lo hizo.

Las emociones que manifiestan los niños en sus historias y en sus dibujos denotan un abanico de emociones que va desde la alegría y felicidad hasta la tristeza, incluso, hay casos en los que los mismos niños manifiestan en la experiencia de migrar ambas emociones.

Por un lado, los niños que manifiestan sentimientos de tristeza, al igual que lo encontrado por Guzmán-Carrillo y GonzálezVerduzco (2013), sienten tristeza, enojo, soledad, abandono y esto no solo sucede con los niños mexicanos; la Red de Migrantes de Nicaragua reporta que $77 \%$ de los niños (as) encuestados expresó sentir tristeza desde que el padre o madre decide irse hasta que regresa. Según estos autores, dichos menores crecen con altos grados de vulnerabilidad emocional, obligándolos a una maduración temprana y con el riesgo de presentar deserción escolar y embarazos prematuros. Así también, LópezPozos, (2009) encontró en un estudio con familias de Tlaxcala y California, sentimientos de tristeza y de abandono manifestándose con rebeldía y desadaptación.

Las cuestiones anteriores se dan, de manera más frecuente, cuando la madre es quien decide migrar, ya que ella es considerada el principal referente afectivo, y resulta ser la figura más importante en el desarrollo emocional del niño (a); esto es cada vez más común, debido al creciente número de mujeres migrantes (Liwski, 2017). Sin embargo, para el caso de los niños de este estudio, no se preguntó a todos si su familiar migrante directo era su padre o madre, por lo cual no se podría expresar un juicio concluyente al respecto.

En este estudio, se enfatizan las similitudes entre la tristeza y la felicidad que expresan los niños ante la migración. Por su parte, la mayoría de los estudios encontrados resaltan el efecto y la apreciación negativa que tiene el niño sobre este fenómeno (Gamarra et al., 2006; García \& Sanz, 2002; Maldonado, 2005, citados por Vera, 2009; Esquivel, 2010), y pocos hablan de manera favorable sobre lo que experimentan los niños al interpretar como un logro el que sus familiares migrantes crucen al otro lado (Moctezuma, 2012, citado por Rivera-Heredia \& Rayo, 2015) y poder recibir beneficios de algún tipo ante dicha situación (Zapata, 2009).

Los niños de este estudio muestran emociones de alegría y felicidad por la migración de un familiar, a pesar de que se enfrenten a diversos peligros y experiencias desagradables, tanto los que se van como los que se quedan. Desde la perspectiva de la resiliencia, se podría decir 
que estos niños, de alguna manera, muestran su resiliencia y la de sus familiares, que aun en la adversidad, puede rescatar lo positivo que tiene tal circunstancia (González-Arratia, ValdezMedina \& Van-Barneveld, 2009; Castañer, 2017).

Por otro lado, hay niños que expresan o manifiestan tanto emociones simultáneas de alegría como de tristeza ante la migración de un familiar; en sus escritos consideran que la migración es tanto buena como mala o expresan verbalmente que la migración es mala, pero en sus dibujos muestran rostros alegres o viceversa; más que interpretar esto como una contradicción en los niños, se confirma que el niño vive tanto emociones agradables como desagradables ante la migración de un familiar. Ojeda, Bailón y García (2014), hablan de esa posibilidad del niño de reconocer o tratar de equilibrar aquellas emociones que podrían parecer contradictorias, pero que ambas son parte de la experiencia al tener un familiar migrante.

También se da el caso de niños que solo ven la migración como algo malo, debido a que sus familiares se alejan de ellos, los dejan solos; mientras que para otros niños, la migración representa algo bueno, ya que permite a los familiares que están lejos venir a visitarlos y proveerlos de recursos materiales. Este hecho hace visible la percepción ambivalente de los niños ante un mismo evento, y a partir de ello surge la pregunta sobre ¿qué lleva a estos niños a percibir la migración de una u otra manera, o de ambas?, y ¿cómo esta percepción tiene relación con el propio desarrollo socioemocional? Preguntas que podrían ser contestadas en estudios posteriores.

Con relación a la capacidad empática, de acuerdo a Piaget, ésta se da principalmente en el periodo formal de la inteligencia. En este estudio, se observa que los niños desde los 9 años muestran empatía hacia otras personas que viven la migración directa o indirectamente, es decir, contrario a lo que propone Piaget, estos niños presentan la capacidad de ponerse en el lugar del otro en edades más tempranas, como lo manifiestan también Oros y Fontana (2015), al igual que Rodas (2016).

Respecto al desarrollo moral, se observó que niños menores de 12 años juzgan la migración como buena o mala en la medida que se sienten afectados personalmente o sus seres cercanos; por otro lado, los niños de 12 años juzgan la migración de acuerdo con las injusticias que perciben en el contexto sociocultural, es decir, aspectos de orden social, tal como lo propusieron Kolberg y Hers (1977). Sin embargo, hay pocos casos de niños de 8 años que muestren una valoración de la migración con base en el orden social.

De manera general, se podría decir que los niños de este estudio presentan habilidades socioemocionales en edades más tempranas que las propuestas por autores clásicos en el tema.

Asimismo, a partir del análisis de los dibujos se puede observar la diversidad de habilidades, de experiencias y contextos que influyen en el qué y cómo se expresan los niños. Por ejemplo, 
en los estados se pudieron identificar diferencias, de tal forma que los estados del centro y occidente (Zacatecas, Michoacán, Hidalgo, Jalisco) refieren más historias de migración directa; por su parte, los niños de Yucatán se refieren más frecuentemente a una migración de lo rural a lo urbano, por mencionar algunas generalidades. En investigaciones a futuro habría que hacer un análisis más exhaustivo sobre las particularidades de los contextos y de su influencia, tanto en el desarrollo como en la experiencia migratoria.

Por su parte, el hecho de poder reconocer y hablar de las emociones es educación emocional (López-Cassá, 2005; Arias et al., 2012). La educación emocional para todos los niños es necesaria, pero para aquellos con experiencias migratorias lo es por los procesos de adaptación que viven al migrar un familiar y con mayor razón si éste es un progenitor. Por ello, es imprescindible que tanto en el sistema educativo, comunitario y familiar, se atienda al niño y a su vivencia. Justis, Almestro y Silva (2017), refuerzan esta idea al mencionar que al educar en las emociones, se busca minimizar la vulnerabilidad de la persona a determinadas situaciones de repercusión social.

El uso de la narrativa y del dibujo, además de mostrar la complejidad que le van agregando los niños a sus construcciones de acuerdo con su edad, también estos materiales expresan el mundo subjetivo de los niños respecto a sus emociones, sentimientos, pensamientos e ideas sobre el fenómeno de la migración. Así como esta técnica de recolección de datos sirvió para tener un acercamiento a aspectos evolutivos como a la experiencia migratoria de los niños, se podría implementar para comprender lo que le sucede a los niños respecto a otros fenómenos psicosociales.

Es necesario reconocer algunas limitaciones de este trabajo. Aunque se utilizó el método de la narrativa, solo se hizo para que los niños escribieran una historia a partir de su dibujo. Algunos narraron historias cercanas a ellos y otros más situaciones de su medio inmediato, por ende, en estudios posteriores se podría obtener más información si se les pide de manera explícita que cuenten historias en relación con su historia personal con la migración.

Si la historia fuera narrada de manera oral y no escrita, tal vez se obtendría mayor información y se podría profundizar en el fenómeno estudiado (Liamputtong, 2013). Esto se lograría al entrevistar a los niños mientras éstos hacen sus dibujos, empero, al haber aplicado la técnica del dibujo y la narración de la historia de manera grupal, no se permitió ahondar en lo que pasa con estos niños respecto a su experiencia con la migración.

En estudios posteriores, se recomendaría reunir grupos más pequeños o de manera individual, dialogar con mayor profundidad con los niños respecto a sus historias, además de establecer de manera anticipada y más clara los procedimientos para el llenado y el vaciado de 
la información de cada estado, debido a que fue un reto integrar la información en algunos casos donde no se tenían los mismos datos.

Contar la historia propia puede ser liberador. Ayudar a que los niños comprendan sus emociones, los pueden movilizar para conseguir un beneficio o evitar un daño (Mora, 2008). El ejercicio de expresar emociones a través del dibujo y la narrativa, además de servir de diagnóstico en cuanto a cómo están viviendo estas experiencias migratorias los niños y en qué circunstancias, también podría servir como medio de educación socioemocional, donde los niños puedan reconocer y expresar las emociones en torno a sus experiencias migratorias directas o indirectas.

\section{Referencias}

Arias, A. V., Morales, J. F., Nouvilas. E. y Martínez, J. L. (2012). Psicología Social Aplicada. Madrid: Panamericana.

Bordignon, N. (2005). El desarrollo psicosocial de Erick Erickson. El diagrama epigenético deladulto. Revista LasallistadeInvestigación, 2(2), 50-63.

Boss, P. (2001). La pérdida ambigua. España: Gedisa.

Castañer, A. (2017). Migración resiliente, herramientas de rescate emocional para niñas, niños y adolescentes. UNICEF. Recuperado de https://www.unicef.org/ mexico/spanish/VCEMigracionResiliente_ mar2018.pdf
Esquivel, F. (2010). Psicoterapia Infantil con Juegos. Casos Clínicos. México: El Manual Moderno S. A. de C. V.

Falicov, C. (2001). Migración, pérdida ambigua y rituales. Perspectivas Sistémicas, 13(69), $\mathrm{s} / \mathrm{p}$. Recuperado de http://www.redsistemica. com.ar/migracion $2 . h t m$

Garaigordobil, M.y Maganto, C.(2011). Empatía y resolución de conflictos durante la infancia y la adolescencia. Revista Latinoamericana de Psicología, 43(2), 255-266.

García-Ledesma, R. y Suárez-Castillo, M. (2007). La pérdida ambigua: una prolongada aflicción de la familia. Psicología y Ciencia Social, 9(2), 32-41.

Gloria, A. M. (1999). Apoyando estudiantes Chicanas: Therapeutic factors in Chicana college students support groups. Journal for Specialists in Group Work, 24(3), 246-259.

González Arratia, N. I., Valdez Medina, J. L., van Barneveld, H. y González Escobar, S. (2009). Resiliencia y salud en niños y adolescentes. Ciencia Ergo-Sum, 16(3), 247-253.

Gortberg, E. (2008). Resiliencia descubriendo las propias fortalezas. Buenos Aires: Paidós.

Guzmán-Carrillo, K. y González-Verduzco, B. (2013). Recursos psicológicos en niños con familiares migrantes (Tesis de licenciatura no publicada). México: Facultad de Psicología de la Universidad Michoacana de San Nicolás de Hidalgo.

Heras, D., Cepa, A. y Lara, F. (2016). Desarrollo emocional en la infancia. International 
Journal of Developmental and Educational Psychology, 1(1), 67-73.

Justis, O., Almestro, S. y Silva, O. (2017). Pedagogía para el desarrollo socioemocional. A propósito de la gestión de aprendizaje en el contexto santiaguero. Revista Iberoamericana De Educación, 75(2), 109126. Recuperado de https://rieoei.org/RIE/ article/view/2636

Kohlberg, L. y Hersh, R. (1997). Moral Development: A Review of the Theory Lawrence. Theory into Practice, 16(2), 5359.

Liwski, N. (23 de febrero de 2017). Migraciones de niñas, niños, y adolescentes bajo el enfoque de derechos. Recuperado de http:// www.derechosinfancia.org.mx/Documentos/ Migraciones_liwski.pdf

López J. y Scandroglio, B. (2007). La metodología cualitativa en la intervención psicosocial. En A. Blanco y J. Rodríguez (Coords.), Intervención psicosocial (pp. 555606). Madri: Pearson Education.

López-Cassá, E. (2005). La educación emocional en la educación infantil. Revista Interuniversitaria de Formación del Profesorado, 19(3), 153-167.

López-Pozos, C. (2009). El costo emocional de la separación en niños migrantes: un estudio de caso de migración familiar entre Tlaxcala y California. Agricultura, Sociedad $y$ desarrollo, 1(6), 81-103.
Mora, F. (2008). El reloj de la sabiduría. Tiempos y espacios del cerebro humano. Madrid: Alianza.

Ojeda García, A. (2014). El dibujo como expresión del mundo interno del migrante. México: Universidad Iberoamericana.

Oros, L. y Fontana, A. (2015). Niños socialmente hábiles, ¿Cuánto influye la empatía y las emociones positivas? Interdisciplinaria, 32(1), 109-125.

Ortiz, M., López-Sánchez, F., Fuentes, M. y Etxebarria, I. (2014). Desarrollo afectivo y Social. México: Psicología Pirámide.

Palmero Cantero, F., Guerrero, C., Gómez, C. y Carpi, A. (2006). Certezas y controversias en el estudio de la emoción. Revista Electrónica de Motivación y Emoción, 9(23-24), 1-25.

Papalia, D., Wendkons, S. y Duskin, R. (2009). Psicología del desarrollo. De la infancia a la adolescencia. México: McGraw Hill.

Piras, G. (2016). Emociones y migración: Las vivencias emocionales de las hijas y los hijos que se quedan en origen. Psicoperspectivas, 15(3), 67-77.

Puleo, E. M. (2012). La evolución del dibujo infantil. Una mirada desde el contexto sociocultural merideño. Educere, 16(53), 157-170.

Ramos, P. (2012). Aplicación de terapia grupal con enfoque humanista a adolescentes con síntomas de depresión en hijos de padres migrantesenlaUnidadEducativaFiscalMixta Central la Inmaculada (Tesis de maestría). 
Recuperada de http://dspace.uazuay.edu.ec/ bitstream/datos/1791/1/09252.pdf

Reeve, J. (2004). Motivación y emoción. México: McGraw Hill.

Rivera-Heredia, M. E. y Rayo-Varona, E. (2015). Migración y Transnacionalidad desde una mirada infantil. El caso de dos comunidades michoacanas de alta tradición Migratoria. En C. Leco y J. C. L. Chávez-Navarro (Eds.), Migración Vulnerable en Michoacán (7579). México: Editorial Morevallado ININEEUMSNH.

Rodas, B. (2016). La empatía cognitiva y el desarrollo de habilidades sociales en los niños y niñas de 5-6 años de la Unidad Educativa Alfonso Troya (Proyecto de tesis). Ecuador: Universidad Técnica de Ambato.

Serry, T. y Liamputtong, P. (2013). The indepth interviewing method in health. En P. Liamputtong (Ed.), Research Methods in Health: Foundations for Evidence-based Practice (pp. 39-53). Inglaterra: Oxford University Press.

Strauss, A. (1970). Descubriendo nuevas teorías de teorías previas. En Shibutani, T. (comp.), Naturaleza humana y comportamiento colectivo. Ensayos en honor a Helbert Blumer. Nueva Jersey: Prentice Hall.

Vera, J. A. (2009). Depresión, ansiedad y estrés en niños y niñas jornaleros agrícolas migrantes. Psicología y Educación, 40, 337345.
Zapata, A. (2009). Familia transnacional y remesas: padres y madres migrantes. Revista Latinoamericana de Ciencias Sociales Niñez y Juventud, 7(2), 1749-1769.
Recibido: 13/03/2019

Revisado: 18/06/2019

Aceptado: 03/07/2019 